\title{
Robot-assisted hysterectomy: a critical evaluation
}

\author{
This article was published in the following Dove Press journal: \\ Robotic Surgery: Research and Reviews \\ 18 March 2015 \\ Number of times this article has been viewed
}

\section{Paul Buderath \\ Bahriye Aktas \\ Martin Heubner \\ Rainer Kimmig}

Department of Gynecology and Obstetrics, University of DuisburgEssen, Essen, Germany
Correspondence: Rainer Kimmig

Department of Gynecology and

Obstetrics, University of Duisburg-Essen, Hufelandstraße 55, 45 I 47 Essen, Germany Tel +4920I723244l

Email rainer.kimmig@uk-essen.de

\begin{abstract}
The introduction of robot-assisted surgery is one of the most remarkable and controversially discussed technological innovations of the past decade in the field of surgery. The technique has been used by an ever-growing number of surgeons in different surgical disciplines throughout the world. In the field of gynecologic surgery, besides myomectomy, hysterectomy in both benign and malignant conditions is one of the main indications for the use of robot-assisted surgery. The establishment of a robot-assisted approach to hysterectomy has led to an increase in minimally-invasive procedures compared to open surgery, accompanied by a decrease in the use of traditional laparoscopy. However, critics question the practical use of the technology and stress the often significantly longer operating room times as well as raised costs. There is a striking contrast between the emphasis put on possible weaknesses by critics on the one hand and the often uncritical marketing of the technology on the other hand. The purpose of this review is to provide a critical evaluation of the possible advantages and disadvantages of robot-assisted hysterectomy from the surgical, economic and organizational perspective based on the present literature. A Pubmed database search was performed using keywords such as robotic, robot-assisted, hysterectomy, training, benign and malignant. All publications were thoroughly reviewed regarding their ability to help evaluate the major benefits and challenges of robot-assisted hysterectomy compared to open or conventional laparoscopic procedures and to determine the current and possible future status of robotic surgery in the performance of hysterectomies.
\end{abstract}

Keywords: robot-assisted surgery, laparoscopic surgery, gynecological surgery

\section{Introduction}

The introduction of robot-assisted surgery is one of the most remarkable and controversially discussed technological innovations of the past decades in the field of surgery. Starting with the approval of the daVinci ${ }^{\circledR}$ Surgical System (Intuitive Surgical, Inc., Sunnyvale, CA, USA) by the United States Food and Drug Administration (FDA) in 2000, the technique has since been used by an ever-growing number of surgeons in different surgical disciplines throughout the world. ${ }^{1}$ The technique represents a further development in laparoscopic surgery, during which the instruments are navigated by the surgeon using control masters, while sitting at a console providing three-dimensional, high-definition vision. The major advantages of robot-assisted surgery are the three-dimensional vision, increased flexibility of the wristed instruments controlled by the surgeon, and ultraprecise motion control.

The US FDA approval for gynecologic surgery followed in $2005 .{ }^{1}$ In the field of gynecologic surgery, besides myomectomy, hysterectomy in both benign and malignant conditions is one of the main indications for the use of robot-assisted surgery. This is not surprising, bearing in mind that hysterectomy is the most common nonobstetric 
major procedure performed in women, with over 500,000 procedures being performed in the US per year. ${ }^{2}$ Hysterectomy is the treatment of choice for early cervical cancer, as well as for endometrial cancer. ${ }^{3,4}$ Depending on the type and stage of the disease, the procedure will be performed as radical or modified hysterectomy. In many cases, radical hysterectomy will be carried out together with therapeutic lymph node dissection of the pelvic and/or para-aortic department. The traditional approach is still open abdominal surgery. However, even in malignant conditions, a robotic approach to radical hysterectomy and lymphadenectomy has been established by a growing number of gynecologic surgeons throughout the world. ${ }^{5}$

The establishment of a robot-assisted approach to hysterectomy has led to an increase in minimally-invasive procedures when compared to open surgery, accompanied by a decrease in the use of traditional laparoscopy. ${ }^{6}$ Reduced blood loss, faster recovery time, and a lower conversion rate to abdominal surgery are the claimed major advantages of robotic surgery, the differences being most striking in obese patients. ${ }^{7}$ However, critics question the practical use of the technology and stress the often significantly longer operating room times, as well as the raised costs, ${ }^{8-10}$ resulting from the use of robotically-assisted surgery techniques. There is a striking contrast between the emphasis put on possible weaknesses by critics on the one hand, and the often uncritical marketing of the technology on the other hand. ${ }^{11}$ The purpose of this review is to provide a critical evaluation of the possible advantages and disadvantages of robot-assisted hysterectomy from surgical, economic, and organizational perspectives based on the present literature.

\section{Methods}

A PubMed database search was performed using keywords such as "robotic", "robot-assisted", "hysterectomy", "training", "benign", and "malignant". All publications were thoroughly reviewed regarding their ability to help answer the central questions we have identified as crucial in defining the role of robot-assisted hysterectomy:

1. What are the major benefits of robot-assisted hysterectomy compared to open or conventional laparoscopic procedures?

2. What are the major challenges or disadvantages of the procedure?

3. What is the current and possible future status of robotic surgery in the performance of hysterectomies?

We decided to evaluate these questions regarding hysterectomy in benign and malignant conditions separately in order to pay respect to the different demands of the procedures that directly influence the evaluation of robotassisted surgery of these procedures.

\section{Review \\ Opening remarks}

The current role of robotic surgery for hysterectomy has been addressed in a number of publications since its implementation into everyday practice. A total of 32 original publications were selected for inclusion into this review. However, only two randomized controlled trials could be identified. We will review the literature on robot-assisted hysterectomy for benign and malignant conditions separately.

\section{Hysterectomy in benign conditions}

Smorgick et $\mathrm{al}^{6}$ showed that the increased use of robotic techniques for the performance of hysterectomies results in fewer open abdominal procedures. Analyzing the patient records of 673 patients from a single institution who underwent hysterectomy via laparoscopic, robotic, laparotomy, mini-laparotomy, and vaginal approaches during a 6-year time-period, the authors could demonstrate a decrease in hysterectomies performed via laparotomy when comparing the early versus late periods of the study's time window (17.7\% versus $5.5 \%$, respectively). During the same period, the proportion of cases treated using robotic surgery increased from $28.3 \%$ to $64.1 \%$. This led to an overall increase in the rate of patients treated using any minimally-invasive technique $(82.3 \%$ in the early period versus $94.6 \%$ in the late period). In a publication on the current status of robotic hysterectomy, Dubeshter et al ${ }^{12}$ reported significantly shorter hospital stays for robotic, as well as conventional laparoscopic and vaginal hysterectomy when compared to open abdominal surgery.

Few studies have compared robot-assisted hysterectomy with conventional techniques using a randomized controlled study design. One such study comes from Paraiso et al, ${ }^{13}$ who performed hysterectomies on 53 women who were randomly assigned to one of two groups: either receiving conventional laparoscopic hysterectomy or robotically-assisted hysterectomy using the daVinci Surgical System. The authors reported no significant differences concerning the intra- and postoperative complications or postoperative pain and returning to daily life activities. However, skin-to-skin time as well as total operative room time were significantly longer in the robotic group (77 minutes longer skin-to-skin time and 72 minutes longer total operative room time). In a similar approach, Sarlos et $\mathrm{al}^{9}$ performed hysterectomy on 95 women between 2008 and 2011, assigning the patients to a robotic 
or a conventional laparoscopic group by randomization. The findings were similar to that of the study published by Paraiso et al, ${ }^{13}$ with parameters such as blood loss, complications, analgesic use, and return to activity showing no significant differences between groups. However, the gain in preoperative to postoperative quality of life was significantly higher in the robotic group. Again, significantly longer operative times were observed in the robotic group when compared to the conventional group (106 minutes versus 75 minutes, respectively).

A number of publications focus on the use of robotic surgery for obese and morbidly obese women. Evaluating a sample of 114 morbidly obese women (body mass index [BMI] $\geq 30$ ), Geppert et $\mathrm{al}^{7}$ reported significantly shorter inpatient times, reduced blood loss, and lower complication rates when comparing robotic and open abdominal hysterectomy. However, the authors also showed significantly longer operative times in the robotic group, though this effect disappeared when evaluating women whose BMI was higher than 35 .

In a prospective, matched case-control study, Sarlos et $\mathrm{al}^{1}$ compared 40 women who underwent robotic hysterectomy with matched controls receiving total laparoscopic hysterectomy, finding similar results to the studies mentioned earlier, such as comparable outcome data and longer operative times in the robotic group. However, a cost analysis was also performed, showing significantly higher operative costs for the robotic procedure (€4,067 versus $€ 2,151)$. A similar observation was made by Rosero et al, ${ }^{14}$ who found costs for robotic hysterectomy to be on average US\$2,489 higher when compared to laparoscopy.

In a thorough economic analysis of robotic surgery for hysterectomy in benign conditions, as well as in patients with endometrial cancer, including 180,230 women undergoing hysterectomy in the US between 2006 and 2012, Wright et $\mathrm{al}^{10}$ examined the influence of surgeon and hospital volume on costs of the procedure. Surgeon and hospital volumes were defined as the number of surgeries performed before the index surgery. While reporting significantly higher unadjusted median costs of robotic surgery compared to conventional laparoscopic hysterectomy (US\$8,152 versus US\$6,535), the authors also found a substantial decrease in the cost difference with growing surgeon and hospital volumes, indicating the role of surgeon experience and the number of procedures performed on the realization of cost effectiveness for robotic surgery. However, in all scenarios calculated by Wright et al, ${ }^{10}$ the costs remained significantly higher in the robotic group.
When discussing the increased costs of robotic procedures, Barbash and Glied ${ }^{15}$ have raised the question of whether the new technology influences patients, as well as surgeons, to choose surgical instead of nonsurgical treatment options, just by looking at the short-time benefits of surgical therapy.

Few authors have investigated the implications of robotic surgery on the surgical training of fellows. The daVinci System offers the possibility of using a second surgical console, allowing a second physician to view the same three-dimensional picture as the primary surgeon. Instrument control can be handed over from one surgeon to the other. Smith et $\mathrm{al}^{16}$ analyzed 222 patients who underwent either dual-console robotic or classical laparoscopic gynecologic surgery between 2009 and 2010. The authors demonstrated similar complication rates and outcomes between cohorts. The estimated blood loss, as well as the total operative time, was significantly reduced in the dual-console robotic group.

An aspect that has hardly been addressed in the literature so far is the effect of a robotic surgical approach on the surgeon. The ergonomics of surgeons' movements and the incidence of musculoskeletal problems are certainly interesting points. A recent study by Sari et $\mathrm{al}^{17}$ showed that $73 \%$ of laparoscopic surgeons reported neck, back, shoulder, or hand pain during or after laparoscopy. The issue has been addressed by Zihni et al, ${ }^{18}$ who performed electromyography measurements of bilateral biceps, triceps, deltoid, and trapezius muscles on a surgeon performing classic laparoscopic as well as robotassisted procedures. The authors reported significantly lower muscle activation during the robotic procedures, perhaps hinting at a more ergonomic way of working.

\section{Radical hysterectomy in gynecologic oncology}

In 1992, Nezhat et al ${ }^{19}$ described the first laparoscopic radical hysterectomy. Since then, it has largely been accepted that a laparoscopic approach is feasible and safe, and that it results in reduced blood loss as well as shorter hospital stays. ${ }^{20-22}$ Still, the minimally-invasive approach has not been established widely due to the high level of training and operative skills needed to perform complex surgical procedures laparoscopically, resulting in a slow learning curve.

Unsurprisingly, a robotic approach has also been used for the performance of radical hysterectomies. In a 2008 survey among their members, the Society of Gynecologic Oncology (SGO) found that $24 \%$ of the respondents already used robotic surgery in gynecologic oncology, with $66 \%$ planning to increase their use of the procedure in the future. ${ }^{5}$ 
Maggioni et $\mathrm{al}^{23}$ compared a cohort of 40 patients who underwent robot-assisted radical hysterectomy in early cervical cancer with a historic cohort of 40 patients who underwent the traditional laparoscopic procedure. It was shown that blood loss and length of stay differed significantly between the groups, in favor of the robotic group. However, the operative time was significantly shorter in the conventional laparoscopic group than in the robotic group (199.6 minutes versus 272.27 minutes, respectively), and there were significantly less lymph nodes removed in the robotic group than the conventional laparoscopic group (20.4 versus 26.2 , respectively). There was no significant difference in terms of postoperative complications between groups. However, the study did not report on oncological outcomes due to the short observation time.

Bogess et $\mathrm{al}^{24}$ reported on the outcomes of 51 consecutive patients who underwent robot-assisted hysterectomy compared with the outcomes of 49 patients who underwent open radical hysterectomy for early-stage cervical cancer. The authors found significantly less blood loss, shorter operative times, and better lymph node retrieval in the robotic cohort. ${ }^{24}$

Hoogendam et $\mathrm{al}^{25}$ reported on the oncological outcomes and on the long-term complication rates of a total of 104 women treated by robotically-assisted radical hysterectomy for cervical cancer with an International Federation of Gynecology and Obstetrics (FIGO) grade of IA1-IIB in the years 2008-2013. The study was described by the authors as being the largest single institutional cohort to date of consecutive cases treated by robot-assisted laparoscopy for early-stage cervical cancer. Within a medium follow-up time of 29.5 months, 13 recurrences were observed, and the overall 5-year progression-free survival (PFS) and disease-specific survival rates were $81.4 \%$ and $88.7 \%$, respectively. The findings suggest that the oncological outcomes of robotically-treated cervical cancer patients are comparable to those of patients being treated by open abdominal surgery. ${ }^{26,27}$ However, the group also reported on relevant long-term complications such as lymphedema (26\%), lower urinary tract symptoms (19\%), urinary tract infection (17\%), and sexual disorders (9\%).

In a retrospective analysis, Cantrell et $\mathrm{al}^{28}$ assessed PFS and overall survival in 63 women with cervical cancer who underwent type 3 robotic radical hysterectomy between 2005 and 2008, comparing the results to a historical cohort of patients who underwent open radical hysterectomy at the same institution between 1995 and 2007. After a median follow-up time of 12.2 months, the authors reported one recurrence and death from cervical cancer, resulting in both a PFS and OS of $96 \%$, hinting at an oncologic outcome that

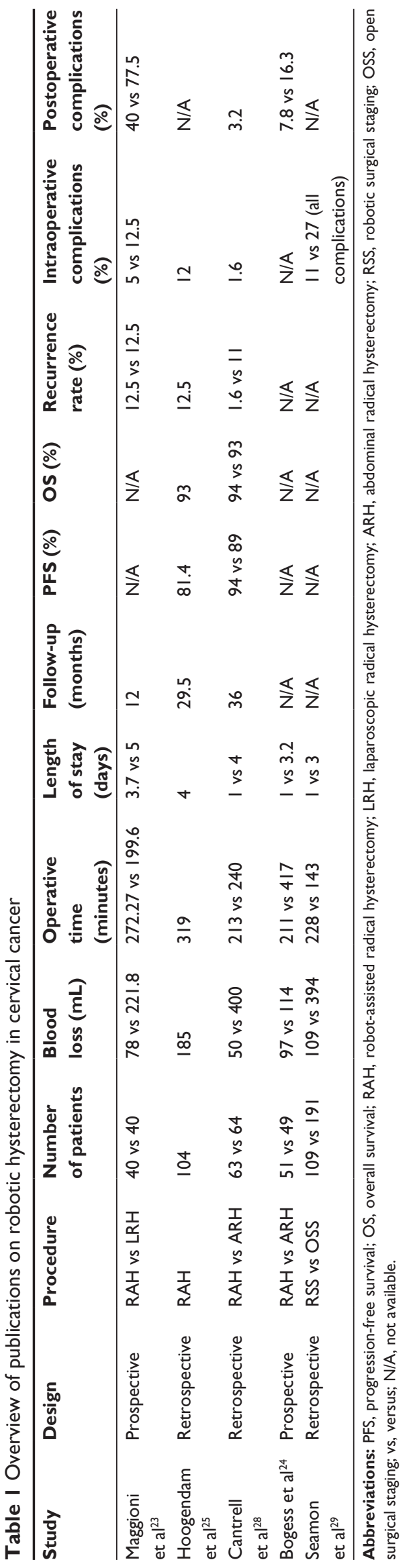


was at least equivalent to the historic cohort, even though the authors mentioned the short observation time as a possible limitation. The complication rate was lower in the robotic group (two versus three serious postoperative complications). The authors also described reduced blood loss $(50 \mathrm{~mL}$ versus $400 \mathrm{~mL}$ ), shorter hospital stays (1 day versus 4 days) and, curiously, even shorter skin-to-skin time (213 minutes versus 240 minutes) in the robotic group when compared to the open hysterectomy group, respectively.

When comparing robotic to open abdominal surgery for surgical cancer staging in obese women $(\mathrm{BMI}>30)$, Seamon et $\mathrm{al}^{29}$ found similar results, with blood loss $(109 \mathrm{~mL}$ versus $394 \mathrm{~mL}$ ), length of stay (1 day versus 3 days), and complication rates (11\% versus $27 \%$ ) being significantly lower in the robotic group when compared to the open abdominal surgery group, respectively. However, the authors also reported a significantly longer operative time (228 minutes versus 143 minutes) in the robotic group. The results from the mentioned studies are summarized in Table 1.

A point worth investigating is the role of robotic surgery in the minimally-invasive performance of even more demanding oncological procedures. In 2001, the compartment-based approach to radical hysterectomy in cervical cancer patients was first described by $\mathrm{Höckel}^{30}$ as total mesometrial resection (TMMR). We described the translation of the technique to robotic surgery, as well as its adoption for endometrial cancer patients known as peritoneal mesometrial resection. ${ }^{31,32}$ In our own cohort of 26 patients with stage IA-IIB cervical cancer treated by robotic TMMR and therapeutic lymphadenectomy, we were able to show the feasibility and safety of the procedure. ${ }^{31}$ No transition to open surgery was necessary and no intraoperative complications were noted. The postoperative complication rate was $23 \%$. Within a mean follow-up time of 18 months, we noted one distant but no locoregional recurrence of cervical cancer. There were no deaths from cervical cancer during the observation period.

The advantages of robotic surgery in the treatment of gynecologic malignancies have been acknowledged by the SGO in their 2012 statement on the matter. ${ }^{33}$ The group recommended that fellows should be trained in both robotic and classic laparoscopic surgical methods, highlighting the technology's potential for more widespread use in the future, while mentioning the operative costs as a potential barrier to the acceptance of the method.

\section{Discussion}

Reviewing the literature in the field shows that a robotic approach to hysterectomy for both benign and malignant conditions has been adopted by surgeons in a large number of countries. However, the question has not been sufficiently answered yet, as to which cases and to what extent the new technology benefits the patients as well as the surgeon.

As was pointed out, robotic surgery is generally characterized by enhanced, three-dimensional vision, more precise instrument control and flexibility, as well as improved ergonomics when compared to classic laparoscopy. On the other hand, most publications report longer operative times, as well as significantly higher costs. ${ }^{6,11,21,27}$ Complication rates and length of stay seem to be comparable, as are outcomes in oncologic cancer patients.

These findings suggest no real benefit of robotic surgery at first sight. However, a closer look should be taken at the data reported. Most comparisons refer to classic laparoscopic surgery. As mentioned earlier, Smorgick et $\mathrm{al}^{6}$ showed that the use of robotics reduces the number of open abdominal procedures. It is evident from these observations that the enhanced possibilities of robot-assisted surgery allow the surgeon to even perform complex procedures using a minimally-invasive approach, thus avoiding laparotomy. It seems obvious that at least in these patients, robotic surgery helps reduce complications and blood loss, as well as length of stay and, arguably, also costs. One could argue that it is possible to obtain the skills to perform most surgeries using conventional laparoscopy by intensive training, thus making the use of robotics unnecessary. While it may be questioned if this is true or not for the majority of gynecologic surgeons, there can be little doubt that robotic technology enables a much larger number of surgeons to perform complex procedures in a minimally-invasive way. This has been proven to be beneficial to patients, as was mentioned earlier.

Robotic surgery is often described as offering advantages, especially for the growing population of obese and morbidly obese patients - a group of patients that faces significantly increased postoperative risks when undergoing laparotomy for gynecologic procedures. ${ }^{34,35}$ In these patients, classic laparoscopy often meets its limits, resulting in conversion to primary laparotomy. As shown by Geppert et al, ${ }^{7}$ the otherwise prolonged operative time when performing robotically-assisted hysterectomy as compared to open abdominal surgery did not differ between groups when the patients' BMI exceeded 35 . This hints at the improved operability that robotic surgery provides in these high-risk patients, offering a minimallyinvasive approach when classic laparoscopy could not. Again, it is likely that a relevant percentage of gynecologic surgeons would choose primary laparotomy in these patients when there was no robot-assisted approach available. 
A point often highlighted by critics of robot-assisted surgery is the increased cost of the procedure. This has been well documented in a number of publications, as previously stated. ${ }^{1,10,14}$ These costs result partly from longer operative times, partly from material costs, and even from anesthesia induction and draping. ${ }^{36}$ However, the findings of Wright et $\mathrm{al}^{10}$ are encouraging, showing that costs can be decreased to a certain degree by raising hospital and surgeon volumes. It does not seem surprising that a higher number of surgeries performed at an institution helps to optimize management, and that the growing experience of the surgeon leads to the more effective use of resources, as well as to shorter operative times resulting in reduced costs. It must not be forgotten that robotic surgery is still a very young technology. As with all technological innovations, costs are high in the beginning. In our opinion, it can be expected that more widespread use will lead to a general decrease in costs, especially since the future introduction of different robotic surgical systems to the market will open the competition for more cost-effective solutions.

Looking at hysterectomy for malignant indications, the feasibility of even the most complex oncosurgical procedures has been demonstrated in a number of publications..$^{21-23,29,30}$ Though even radical hysterectomies and lymphadenectomy can be performed by classic laparoscopy, the benefits of robotic surgery, such as increased motion range, an enhanced three-dimensional view, and ultraprecise instrument control, make complex procedures a lot easier to learn, thus offering a minimally-invasive approach to a larger number of patients. This is especially evident in the translation of complex, compartment-based surgical procedures such as TMMR to robotic surgery, as shown by our group. ${ }^{31}$

Along with the implementation of new techniques, the training of fellow surgeons changes. In addition, the feasibility of dual-console robotic training has been demonstrated by Smith et al. ${ }^{16}$ It seems likely that the increased visibility of anatomic structures contributes to a better understanding of surgical steps, compared to the sometimes limited vision in open abdominal surgery, as well as to the two-dimensional picture of classic laparoscopy. Moreover, the technology also allows continuous video recording of surgical procedures, which can be used for documentation as well as for educational purposes. It appears to be convincing that this also facilitates the definition of surgical standards, especially for complex oncological procedures, such as the compartmentbased approaches mentioned herein.

Last, but not least, the results of Zihni et a ${ }^{18}$ hint at possible benefits for the surgeon resulting from improved ergonomics - an issue that should be taken into account in order to avoid long-term complications in laparoscopic surgeons, such as musculoskeletal symptoms. This is especially relevant with regard to long-lasting complex procedures in gynecologic oncology.

\section{Conclusion}

The review of the literature on robot-assisted hysterectomy clearly shows that the usage of this method is on the rise throughout the world. However, the extent to which the procedure is beneficial to both surgeons and patients remains unclear. It seems obvious from the reported data that the uncritical use of robotics for procedures that can be easily performed via classic laparoscopy does not harm the patient, as outcome variables were shown to be at least equally as good; however, the use of robotics does raise costs and prolongs the operative time significantly. On the other hand, there can be no doubt that a robotic approach to hysterectomy can be very useful in selected cases. In these situations - namely, in complex oncological procedures, procedures performed with obese patients, or difficult surgical conditions - the use of the robot helps avoid laparotomy and thus benefits the patient substantially. However, a sufficient number of cases is needed for economic reasons, as well as for gaining experience and expertise. Thus, it can be adequate to perform even less complex procedures using a robotic surgical system in specialized institutions.

As we pointed out earlier, robotic surgery also has the potential to improve the training of fellow surgeons, as well as to define operative standards. In any case, robotassisted surgery is a technology full of potential for future developments. Hopefully, ongoing improvement of the technology will provide robotic surgeons with more affordable and even easier-to-use systems. Combined with the growing experience of robotic surgeons throughout the world, this would help address the still critical issues of costs and operative time.

\section{Disclosure}

The manuscript has been approved by all authors. We declare that there are no conflicts of interest. Rainer Kimmig has received expense allowances/honoraria from Intuitive Surgical Inc. in 2011 to 2014 for educational work in robotic surgery and training of gynecologic departments in Europe (proctoring).

\section{References}

1. Sarlos D, Kots L, Stevanovic N, Schaer G. Robotic hysterectomy versus conventional laparoscopic hysterectomy: outcome and cost analyses of a matched case-control study. Eur J Obstet Gynecol Reprod Biol. 2010; 150(1):92-96. 
2. Jacoby VL, Autry A, Jacobson G, Domush R, Nakagawa S, Jacoby A. Nationwide use of laparoscopic hysterectomy compared with abdominal and vaginal approaches. Obstet Gynecol. 2009;114(5):1041-1048.

3. Wright JD, Barrena Medel NI, Sehouli J, Fujiwara K, Herzog TJ. Contemporary management of endometrial cancer. Lancet. 2012;7;379(9823):1352-1360

4. Roque DR, Wysham WZ, Soper JT. The surgical management of cervical cancer: an overview and literature review. Obstet Gynecol Surv. 2014;69(7):426-441.

5. Mabrouk M, Frumovitz M, Greer M, et al. Trends in laparoscopic and robotic surgery among gynecologic oncologists: A survey update. Gynecol Oncol. 2009;112(3):501-505.

6. Smorgick N, Patzkowsky KE, Hoffman MR, Advincula AP, Song AH, As-Sanie S. The increasing use of robot-assisted approach for hysterectomy results in decreasing rates of abdominal hysterectomy and traditional laparoscopic hysterectomy. Arch Gynecol Obstet. 2014;289(1):101-105.

7. Geppert B, Lönnerfors C, Persson J. Robot-assisted laparoscopic hysterectomy in obese and morbidly obese women: surgical technique and comparison with open surgery. Acta Obstet Gynecol Scand. 2011;90(11):1210-1217.

8. Sarlos D, Kots LA. Robotic versus laparoscopic hysterectomy: a review of recent comparative studies. Curr Opin Obstet Gynecol. 2011;23(4):283-288.

9. Sarlos D, Kots L, Stevanovic N, von Felten S, Schär G. Robotic compared with conventional laparoscopic hysterectomy: a randomized controlled trial. Obstet Gynecol. 2012;120(3):604-611.

10. Wright JD, Ananth CV, Tergas AI, et al. An economic analysis of robotically assisted hysterectomy. Obstet Gynecol. 2014;123(5): 1038-1048.

11. Schiavone MB, Kuo EC, Naumann RW, et al. The commercialization of robotic surgery: unsubstantiated marketing of gynecologic surgery by hospitals. Am J Obstet Gynecol. 2012;207(3):174. e1-e7.

12. Dubeshter B, Angel C, Toy E, Thomas S, Glantz JC. Current role of robotic hysterectomy. J Gynecol Surg. 2013;29(4):174-178.

13. Paraiso MF, Ridgeway B, Park AJ, et al. A randomized trial comparing conventional and robotically assisted total laparoscopic hysterectomy. Am J Obstet Gynecol. 2013;208(5):368. e1-e7.

14. Rosero EB, Kho KA, Joshi GP, Giesecke M, Schaffer JI. Comparison of robotic and laparoscopic hysterectomy for benign gynecologic disease. Obstet Gynecol. 2013;122(4):778-786.

15. Barbash GI, Glied SA. New technology and health care costs - the case of robot-assisted surgery. N Engl J Med. 2010;363(8):701-704.

16. Smith AL, Krivak TC, Scott EM, et al. Dual-console robotic surgery compared to laparoscopic surgery with respect to surgical outcomes in a gynecologic oncology fellowship program. Gynecol Oncol. 2012;126(3):432-436.

17. Sari V, Nieboer TE, Vierhout ME, Stegeman DF, Kluivers KB. The operation room as a hostile environment for surgeons: physical complaints during and after laparoscopy. Minim Invasive Ther Allied Technol. 2010;19(2):105-109.

18. Zihni AM, Ohu I, Cavallo JA, Cho S, Awad MM. Ergonomic analysis of robot-assisted and traditional laparoscopic procedures. Surg Endosc. 2014;28(12):3379-3384.

19. Nezhat CR, Burrell MO, Nezhat FR, Benigno BB, Welander CE. Laparoscopic radical hysterectomy with paraaortic and pelvic node dissection. Am J Obstet Gynecol. 1992;166(3):864-865.

20. Gil-Moreno A, Puig O, Pérez-Benavente MA, et al. Total laparoscopic radical hysterectomy (type II-III) with pelvic lymphadenectomy in early invasive cervical cancer. J Minim Invasive Gynecol. 2005;12(2): 113-120.
21. Bogani G, Cromi A, Uccella S, et al. Laparoscopic versus open abdominal management of cervical cancer: long-term results from a propensitymatched analysis. J Minim Invasive Gynecol. 2014;21(5):857-862.

22. Yin XH, Wang ZQ, Yang SZ, Jia HY, Shi M. Clinical observation of laparoscopic radical hysterectomy for cervical cancer. Int J Clin Exp Med. 2014;7(5):1373-1377.

23. Maggioni A, Minig L, Zanagnolo V, et al. Robotic approach for cervical cancer: comparison with laparotomy: a case control study. Gynecol Oncol. 2009;115(1):60-64.

24. Boggess JF, Gehrig PA, Cantrell L, et al. A case-control study of robotassisted type III radical hysterectomy with pelvic lymph node dissection compared with open radical hysterectomy. Am J Obstet Gynecol. 2008;199(4):357. e1-e7.

25. Hoogendam JP, Verheijen RH, Wegner I, Zweemer RP. Oncological outcome and long-term complications in robot-assisted radical surgery for early stage cervical cancer: an observational cohort study. BJOG. 2014;121(12):1538-1545.

26. Bodurka-Bevers D, Morris M, Eifel PJ, et al. Posttherapy surveillance of women with cervical cancer: an outcomes analysis. Gynecol Oncol. 2000;78(2):187-193.

27. Lim KC, Howells RE, Evans AS. The role of clinical follow up in early stage cervical cancer in South Wales. BJOG. 2004;111(12): 1444-1448.

28. Cantrell LA, Mendivil A, Gehrig PA, Boggess JF. Survival outcomes for women undergoing type III robotic radical hysterectomy for cervical cancer: a 3-year experience. Gynecol Oncol. 2010;117(2):260-265.

29. Seamon LG, Bryant SA, Rheaume PS, et al. Comprehensive surgical staging for endometrial cancer in obese patients: comparing robotics and laparotomy. Obstet Gynecol. 2009;114(1):16-21.

30. Höckel M. [Total mesometrial resection: nerve-sparing extended radical abdominal hysterectomy]. Zentralbl Gynakol. 2001;123(5):245-249. German

31. Kimmig R, Wimberger P, Buderath P, Aktas B, Iannaccone A, Heubner M. Definition of compartment-based radical surgery in uterine cancer: radical hysterectomy in cervical cancer as 'total mesometrial resection (TMMR)' by M Höckel translated to robotic surgery (rTMMR). World J Surg Oncol. 2013;11:211.

32. Kimmig R, Aktas B, Buderath P, Wimberger P, Iannaccone A, Heubner M. Definition of compartment-based radical surgery in uterine cancer: modified radical hysterectomy in intermediate/high-risk endometrial cancer using peritoneal mesometrial resection (PMMR) by M Höckel translated to robotic surgery. World J Surg Oncol. 2013;11:198.

33. Ramirez PT, Adams S, Boggess JF, et al. Robotic-assisted surgery in gynecologic oncology: a Society of Gynecologic Oncology consensus statement. Developed by the Society of Gynecologic Oncology's Clinical Practice Robotics Task Force. Gynecol Oncol. 2012;124(2):180-184.

34. Pitkin RM. Abdominal hysterectomy in obese women. Surg Gynecol Obstet. 1976;142(4):532-536.

35. Johnson A, Young D, Reilly J. Caesarean section surgical site infection surveillance. J Hosp Infect. 2006;64(1):30-35.

36. Zeybek B, Oge T, Kılıç CH, Borahay MA, Kılıç GS. A financial analysis of operating room charges for robot-assisted gynaecologic surgery: Efficiency strategies in the operating room for reducing the costs. J Turk Ger Gynecol Assoc. 2014;15(1):25-29. 
Robotic Surgery: Research and Reviews

Dovepress

\section{Publish your work in this journal}

Robotic Surgery: Research and Reviews is an international, peer reviewed, open access, online journal publishing original research, commentaries, reports, and reviews on the theory, use and application of robotics in surgical interventions. Articles on the use of supervisory-controlled robotic systems, telesurgical devices, and shared-control systems are

invited. The manuscript management system is completely online and includes a very quick and fair peer review system, which is all easy to use. Visit http://www.dovepress.com/testimonials.php to read real quotes from published authors.

Submit your manuscript here: http://www.dovepress.com/robotic-surgery-research-and-reviews-journal 